\title{
Intégration et qualité dans la transformation de l'espace urbain
}

Integration and Quality in Transformations of Urban Space

Roberta Morelli

\section{(2) OpenEdition}

Journals

Édition électronique

URL : http://journals.openedition.org/crau/557

DOI : $10.4000 /$ crau. 557

ISSN : 2547-5746

Éditeur

Éditions du patrimoine

Édition imprimée

Date de publication : 1 novembre 2012

Pagination : 112-122

ISBN : 978-2-7577-0108-9

ISSN : 1296-4077

Référence électronique

Roberta Morelli, «Intégration et qualité dans la transformation de l'espace urbain », Les Cahiers de la recherche architecturale et urbaine [En ligne], 26/27 | 2012, mis en ligne le 01 novembre 2017, consulté le 19 avril 2019. URL : http://journals.openedition.org/crau/557 ; DOI : 10.4000/crau.557 
Comment la complexité de la réalité contemporaine peut (et doit) changer la manière d'aborder la transformation urbaine? La définition de l'approche multi-scalaire et intégrée proposée ici répond à la volonté de dépasser les limites des analyses sectorielles, à travers la mise au point d'un outil d'évaluation de la qualité des projets urbains et architecturaux.

\section{Intégration et qualité dans la transformation de l'espace urbain}

ROBERTA MORELLI

Le poids de l'urbanisation croissante du début $d u x x^{e}$ siècle à nos jours est confirmé, de façon de plus en plus évidente, par I'intérêt que les débats internationaux les plus récents manifestent concernant les rapports entre les dynamiques de transformation de la ville, les développements de la société contemporaine et les équilibres des différents écosystèmes. L'acte de s'établir dans le territoire a toujours été caractérisé, en Europe, par des facteurs liés à des exigences divergentes : enracinement vs expérimentation, conservation vs transformation, continuité vs évolution représentent les tensions dialectiques qui encore aujourd'hui continuent à influencer l'image, l'identité et la structure de toutes les opérations urbaines $^{1}$. Complexe, la réalité actuelle exige une réflexion spécifique sur le sens et sur la responsabilité que ces transformations peuvent (et doivent) assumer vis à vis du futur de nos villes : comprendre les relations profondes entre environnement naturel et espace bâti représente la condition nécessaire pour fonder une nouvelle conscience du " construire ", capable de remettre au

1. Alessandra Battisti, Fabrizio Tucci, Ambiente e cultura dell'abitare. Innovazione tecnologica e sostenibilità del costruito nella sperimentazione del progetto ambientale, Rome, Dedalo, 2000. 
centre de la recherche scientifique et de la pratique professionnelle la qualité de vie en milieu urbain et la valorisation des ressources disponibles.

\section{Complexité de la ville contemporaine}

L'espace urbain, en tant que " contenu-contenant » défini par la composition multiforme d'éléments naturels et artificiels, constitue l'expression visible la plus significative des processus que les activités humaines ont produit dans le temps. II représente aussi le lieu privilégié où se concentrent en même temps les énergies (physiques, intellectuelles et créatives) et les problématiques (sociales, environnementales et économiques) de la contemporanéité. L'incapacité à comprendre et gérer les dynamiques spatiales et environnementales associées aux développements sociaux et économiques les plus récents a produit de nombreux problèmes qui continuent à affecter, de différentes manières, toutes nos villes : au-delà des effets déjà connus liés à la pollution, à la réduction de la biodiversité et à I'utilisation incorrecte des ressources énergétiques, la mauvaise qualité des espaces publics et la perception d'un sentiment croissant d'insécurité induisent des phénomènes de ségrégation qui favorisent la perte progressive d'une " conscience collective » et l'augmentation conséquente des coûts liés à la résolution de ces problèmes $^{2}$. Mais les déséquilibres économiques qui caractérisent nos réalités urbaines produisent aussi des difficultés d'accès au logement et aux services et de nouvelles formes d'exclusion qui réduisent la cohésion sociale et favorisent d'inévitables conflits. L'asservissement fréquent des politiques urbaines aux intérêts économiques particuliers (et à court terme) contribue, de plus, à l'épuisement des identités locales et favorise les opérations de spéculation. Tout ceci a des conséquences plus ou moins visibles, mais certainement négatives et profondes, en termes d'incapacité à exploiter les potentialités des lieux.

Il est vrai, comme soutient le géographe Jacques Lévy, qu'" on ne peut pas exiger des villes qu'elles résolvent tous les problèmes posés par la société » (Lévy, 2008 ${ }^{3}$ ) ; pour autant, la nécessité de s'interroger sur l'impact que l'activité humaine a sur I'habitabilité d'un espace urbain et sur la qualité de vie des individus qui l'occupent semble de plus en plus urgente. À cet égard, les enseignements d'auteurs comme Lewis Mumford et Jane Jacobs ${ }^{4}$ suggèrent de concentrer I'attention sur les facteurs qui contribuent à rendre une ville vivante. Cette approche reconnaît la qualité d'un espace urbain au-delà de sa simple transformation physique, en identifiant sa valeur, par exemple, dans les rapports entre conception des espaces ouverts et vie collective, entre fonctionnalité des bâtiments et leurs modalités d'usage ou entre système bâti et bien-être des usagers. Une pluralité de facteurs différents conditionne donc la qualité et la vitalité d'un espace urbain à travers les effets induits par la multiplicité d'interactions conséquentes. La complexité de ces relations peut (et doit) être interprétée aujourd'hui comme instrument clé d'une relecture des méthodologies de conception et des méthodologies opérationnelles courantes, afin de garantir la pertinence des configurations spatiales, la durabilité des opérations et la consonance des solutions.
2. Janh Gehl, Lars Gemzoe, Public Spaces: Public Life, Copenhague, Danish architectural Press, 1998. Voir aussi Jan Gehl Vita in città. Spazio urbano e relazioni sociali, Rimini, Maggioli Editore, 1991.
3. Jacques Lévy, "On ne peut pas exiger des villes qu'elles résolvent tous les problèmes posés par la société » dans Agir pour les villes et le territoire. Manifesto italo-francese. Uni(di)versité, (Stefano

Panunzi, Marco Cremaschi, Michel Péraldi, Elio Piroddi, Maurizio Carta, Philippe

Foulquié, Marc Augé, Franco Zagari et al), Rome, Ambassade de France, mai 2008.
4. Jane Jacobs, The Death and Life of Great American Cities, New York, Vintage Books, 1961. 


\section{Transformation de l'espace urbain et Intégration}

Dans le cadre de la réalité contemporaine, la transformation de l'espace urbain se trouve confronté à la nécessité de concilier une pluralité de conditions et $d^{\prime}$ intérêts divers ${ }^{5}$. Le concept d'intégration peut (et doit) assumer donc un rôle stratégique face aux enjeux suivants ${ }^{6}$ (fig.1) :

1) déconnection des "éléments "qui composent la ville,

2) fragmentation des " produits » de la conception des espaces de vie,

3) discontinuité des "processus " de transformation urbaine dans le temps.



Fig. 1. L'intégration des

" composants" de la

transformation urbaine

comme réponse

aux enjeux de la ville

contemporaine
Pour ce qui concerne le premier aspect, on se réfère à l'idée que "malgré une superficielle apparence ordonnée, la plupart des villes contemporaines sont simplement une collection de pièces déconnectées, définies sur deux ou trois échelles " (Salingaros et West, 19997). La déconnection des " parties » de la ville est le résultat de logiques qui ignorent les potentialités des différentes forces d'interaction en jeu et qui restreignent l'intérêt du projet au seul périmètre physique d'intervention et/ou à l'image esthétique de l'« objet » architectural. En revanche, comme soutenu par divers auteurs (Salingaros, Alexander, et autres ${ }^{8}$ ), I'intégrité de l'espace urbain est fonction de certaines " règles de composition ", qui sont typiques des systèmes complexes : " union, diversité, bords, forces, organisation, hiérarchie, interdépendance » sont certains des principes qui règlent la cohérence de ces systèmes et qui demandent de remplacer l'« analyse des parties" par l'étude des interconnexions entre sous-systèmes composants. Les tendances de la pratique de ces dernières années, au contraire, ont soutenu la réduction des connexions et la négation de l'inverse proportionnalité entre intensité d'une force d'interaction et rayon relatif d'action, en produisant une élimination progressive des qualités fractales de l'espace urbain. Les éléments de bord, les interfaces de connexion et les espaces intermédiaires doivent alors assumer un rôle central dans les transformations de la ville contemporaine, en tant que "nervures " portantes et " membranes » interactives, responsables des relations entre formes, individus et flux - qui rendent le système urbain hiérarchique et unitaire.
5. Peter Bosselman, Urban Transformation. Understanding City Design and Form, Washington, Island Press, 2009

6. Jon Lang, Urban Design. A Tipology of Procedures and Products, Oxford, Architectural Press, 2005.
7. Nikos Salingaros, Complexity and Urban Coherence, Journal of Urban Design, vol. 5 (p. 291-316), 2000.

8. N. Salingaros, Principles of Urban Structure, Delft, Delft University Press, 2005. Voir aussi Christopher Alexander, Hajo Neis, Artemis Anninou, Ingrid King, A New Theory of Urban Design, New York, Oxford University Press, 1987 et Christopher Alexander, The Nature of Order, vol. 1, 2, 3, 4, New York, Oxford University Press, 2000. 
Parler d'intégration, en se référant aux " produits » de la conception urbaine, signifie se référer à la nécessité de dépasser les fréquentes disputes relatives à la définition des compétences disciplinaires spécifiques. Urbanisme, paysage et architecture préfigurent toujours de nouveaux scénarii d'évolution, mais les dimensions temporelles auxquelles ils se référent sont différentes, tout comme sont différents les vitesses d'exécution des processus et les outils du projet. Les ambiguïtés produites par ces différences, même dans la variété des contextes géographiques, se traduisent encore trop souvent, dans une séparation rigide d'échelles et dans des fréquents conflits sectoriels. De plus, la fragmentation des compétences, bien que résultant d'importants progrès technologiques, contribue à différencier les langages, en réduisant les possibilités de dialogue entre tous les acteurs impliqués. Dépasser ces divisions signifie promouvoir de nouvelles synergies, capables d'améliorer les liens entre des concepts tels que : architecture et urbanisme, environnement et paysage, établissements et infrastructures, conception et construction, forme et technologie - autant de termes caractérisant toutes les actions d'intervention sur la ville.

La nécessité de conjuguer des intérêts souvent opposés, par rapport à la pluralité des facteurs concernant les dynamiques urbaines, conduit à soutenir une intégration nécessaire ainsi entre les «processus » qui règlent ces transformations, afin d'assurer l'accord et la continuité des opérations dans le temps. Cela signifie limiter le traditionnel conflit entre plan et projet et initier une plus forte interdépendance des interventions à échelles différentes : si d'un côté, en effet, l'architecture risque d'être dominée par des logiques stylistiques qui reconnaissent la qualité, dans la singularité de I'« objet » architectural ou dans la « signature » des starchitectes, de l'autre, l'urbanisme risque d'être conçu en termes purement quantitatifs, comme régulateur de poids politiques et de rentes économiques. Pour réduire ces menaces, il faut garantir la synergie des acteurs en jeu et une coordination efficace des outils de la transformation urbaine.

\section{Qualité de l'espace urbain}

La difficulté à "lire » la qualité urbaine, le risque de réduire la durabilité à un effet de mode et la tendance à concevoir le bâtiment comme expression de l'habileté de l'architecte ou de la puissance du client représentent les déviations les plus courantes vis à vis desquels la recherche en architecture doit promouvoir une révision de principes et de stratégies du projet. À ce propos, la contribution en objet propose de concentrer l'attention sur la qualité de l'espace urbain, considérée dans une acception large du terme, comprenant aussi la perspective de la durabilité.

Bien que ces deux concepts fassent l'objet de spéculations théoriques et critiques depuis longtemps, des réalisations concrètes sont encore largement le fruit d'interventions isolées qui tardent à s'édifier en pratiques diffusées. La volonté d'envisager les principes du développement durable dans une vision plus ample de qualité, découle de la conviction que la demande croissante de meilleures conditions de vie est liée à l'interaction d'une multiplicité des paramètres 
différents, dont l'incidence est beaucoup plus grande que celle que les théories écologiques ou l'infinie série des labels actuels, voudraient nous faire croire. Contrairement à la " mesure » de la valeur des interventions au moyen d'analyses sectorielles et de processus de simplification, il faut se référer à une compréhension plus profonde de la complexité urbaine.

Bien que tenter de donner une définition de qualité soit toujours une opération paradoxale - dans le sens où elle n'est jamais complètement objective - poursuivre une révision méthodologique de ce type peut améliorer les capacités d'intervention dans la ville. On propose donc d'approfondir la notion de qualité, par rapport à une série de thèmes référés, pour facilitation de lecture, aux structures fondamentales suivantes:

- Caractère-Contexte, concernant l'ensemble des facteurs identitaires d'un lieu, sur lesquels fonder et exploiter la relation entre contexte et nouveau paysage urbain ;

- Environnement-Communauté, concernant le système de facteurs environnementaux et sociaux de la ville sur lesquels construire les caractères des espaces ouverts et le sens d'appartenance de l'individu aux lieux et aux communautés ;

- Mobilité-Connexions, concernant l'ensemble des facteurs connectifs de la ville - conditionnant l'accessibilité et la sécurité des lieux - sur lesquels baser la cohérence et l'intégrité du système urbain entier ;

- Projet-Construction, concernant l'ensemble des facteurs spatiaux, technologiques et constructifs, sur lesquels concevoir l'adaptabilité des configurations, le confort des usagers et la pertinence des solutions.

\section{Critères d'une approche intégrée du projet}

La qualité urbaine étant entendue comme capacité à atteindre un équilibre dynamique entre la pluralité de tels facteurs, nous nous concentrons sur l'individuation de "critères " à la base d'une approche intégrée du projet, comme relecture critique des principes et des méthodes associés à la transformation de l'espace urbain. À cette fin, nous proposons la définition de "principes de qualité » - en référence aux structures précédentes - et la déclinaison de ces derniers en " thèmes critiques d'intégration » à échelle urbaine et architecturale (fig. 2). Chacun des principes de qualité est défini comme rapport entre deux termes (pour souligner la perspective de l'interaction), choisis comme mots clés de la contemporanéité. La déclinaison des principes en thèmes critiques - également définis par une relation entre deux termes - permet de relier les mots clés à d'importants arguments des processus de définition des opérations urbaines ou architecturales. Mais chacun de ces thèmes, familier des débats scientifiques et culturels, ne constitue pas, en lui-même, «la problématique » du projet : il la définit seulement en partie. L'unicité d'un projet est plutôt inscrite dans l'ensemble de relations mutuelles et complexes qui relient ces thèmes?.

Dans cette perspective, sont proposées deux matrices de synthèse, qui distinguent la dimension urbaine de celle architecturale, pas seulement en relation avec la différence de dimensions, mais surtout par rapport à la complexité de chaque contexte d'intervention. Sans vouloir réduire la réflexion à une

9. Christopher Alexander, Serge Chermayeff, Spazio di relazione e spazio privato, Milan, II Saggiatore, 1968 
principes de qualité

de l'espace urbain
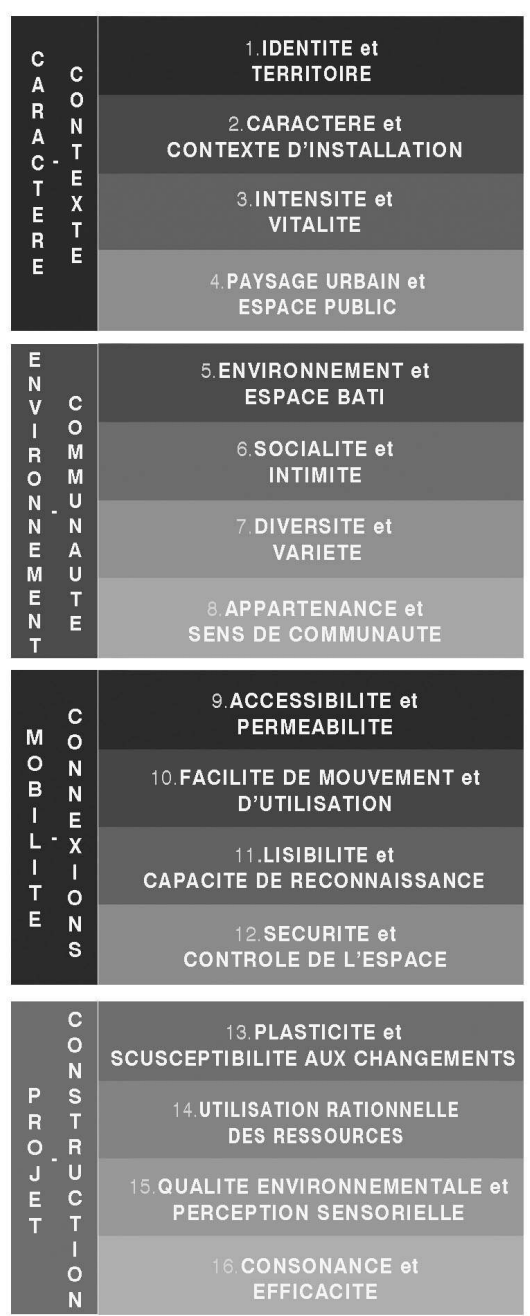

thèmes critiques

d'une approche integrée du projet
a) urbain
b) architectural

1a.utilisation du territoire et
compatibilité des transformations
2a.définition d'échelle et
limites de "charge" urbain
3a.densité et
compacité du tissu bâti
4a.construction du paysage urbain
et espaces ouverts

5a.caractères environnementaux et structure spatiale

6a.espace public et espace privé

7a.aménagement à usage mixte et
complementarité des activités
8a.concertation et
équité sociale

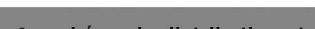

a. schéma de distribution et facilité de mouvement

10 a mobilité et réseaux de connexion

11a.clarté d'implantation et lisibilité de la structure urbaine

12a.liberté de mouvement et contrôle de l'espace

13a.résilience de la forme urbaine
et élasticité de sa structure
1b.contexte d'installation et
vocation identitaire

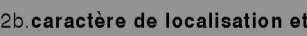
programme d'intervention

3b. modèle d'installation et schéma typologique

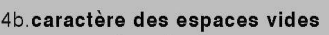
et contexte

$\begin{gathered}5 b . \text { système du vert et } \\ \text { système bâti }\end{gathered}$
$\begin{gathered}6 \mathrm{~b} \text {. espace collectif et } \\ \text { espace individuel }\end{gathered}$
$7 \mathrm{~b}$. mixité des fonctions et
variété des solutions typologiques
$8 \mathrm{~b}$.contact entre les individus
et cohésion sociale

$\begin{gathered}9 \mathrm{~b} . \text { accessibilité et } \\ \text { continuité des parcours }\end{gathered}$
$\begin{gathered}10 \mathrm{~b} . \text { système distributif et } \\ \text { facilité d'utilisation spatiale }\end{gathered}$
$\begin{gathered}11 \mathrm{~b} . \text { cohérence du modèle architectural } \\ \text { et orientation spatiale }\end{gathered}$
$\begin{gathered}12 \mathrm{~b} . \text { sécurité et } \\ \text { habitabilité }\end{gathered}$

$\begin{gathered}\text { 13b.flexibilité et } \\ \text { adaptabilité }\end{gathered}$
$\begin{gathered}\text { 14b.contrôle bioclimatique et } \\ \text { économies d'énergie }\end{gathered}$
15b.confort environnemental
intérieur et qualité des matériaux
16b.coordination operationnelle
et pertinence du projet

Fig. 2. Déclinaison des principes de qualité de l'espace urbain en thèmes critiques de l'intégration à niveau urbain et architectural. 
correspondance univoque entre principes de qualité et thèmes d'intégration, les matrices sont conçues comme un outil d'évaluation multicritères, qui permet d'interpréter la valeur d'une opération comme résultat de la combinaison de différents avantages, grandissant avec la quantité et l'intensité des relations mutuelles " activées ». À cette fin, ces schémas mettent en évidence les interactions prédominantes entre les thèmes critiques définis, soit au niveau urbain (fig. 3) soit architectural : I'incidence réciproque entre deux thèmes est signalée par un point coloré qui permet une lecture horizontale ou verticale du même lien. Chacune de ces mises en correspondance - comme déjà noté pour les principes et les thèmes critiques - ne constitue pas le « cœur » du projet. En effet, chaque interaction entre thèmes est déjà reconnue comme problématique bien identifiée dans les débats actuels; en revanche, l'ensemble des effets produits par la pluralité de ces associations signale un projet à travers sa nature unique et singulière. Dans cette logique, I'attention des acteurs engagés dans un processus, urbain ou architectural, ne doit pas se concentrer sur l'analyse des problématiques singulières; il s'agit plutôt de déterminer, entre les plusieurs séries de "mosaïques » d'interactions possibles, le " point d'équilibre » le meilleur, à l'égard du contexte géographique, environnemental et socioculturel spécifique. Si la transformation urbaine est identifiée par la pluralité des effets simultanés induits par les interactions repérées, sa qualité doit être reconnue dans la cohérence de l'ensemble de ces interactions. La construction des matrices ne tend pas à associer à chaque projet/intervention une « note » ou une catégo- rie d'appartenance, mais veut souligner la complexité des phénomènes en jeu, en suggérant des parcours de réflexion ouverts. Il s'agit donc d'une contribution critique utile, soit pour l'évaluation de l'efficacité des réalisations, soit pour le contrôle, en phase de conception et d'exécution, des potentialités d'un lieu et de ses transformations. À chacune de ces relations n'est pas expressément associé, de plus, un "poids spécifique », dans la conviction que l'évaluation de l'importance relative ne peut pas être le fruit d'un jugement objectif à priori, mais doit faire l'objet d'une étude précise, capable de "faire apparaître » les priorités dans chaque contexte.

\section{Tendances des expériences européennes de projet}

Pour effectuer une vérification expérimentale de cette approche, les matrices ont été utilisées comme bases de l'analyse critique et de l'évaluation comparative de certaines études de cas, référées à 6 villes dans autant de pays européens (Suède, Royaume-Uni, Allemagne, Pays-Bas, France et Italie) : il s'agit de projets finalisés ou en cours de réalisations, sélectionnés pour leurs objectifs affirmés de durabilité, mais surtout pour la recherche d'une cohérence entre stratégies générales et choix spécifiques des réalisations architecturales. Dans chaque ville, un projet urbain et un projet architectural de détail ont fait l'objet d'une analyse basée sur les thèmes critiques d'intégration correspondants, définis dans les deux matrices. L'objectif prioritaire de l'étude a été celui d'offrir un instrument d'approfondissement des caractères d'une approche intégrée, en vérifiant la 
thèmes critiques de l'intégration: projet urbain

1a. $\begin{aligned} & \text { utilisations du territoire et } \\ & \text { compatibilité des transformations }\end{aligned}$
2a. définition d'échelle et
limites de "charge" urbain
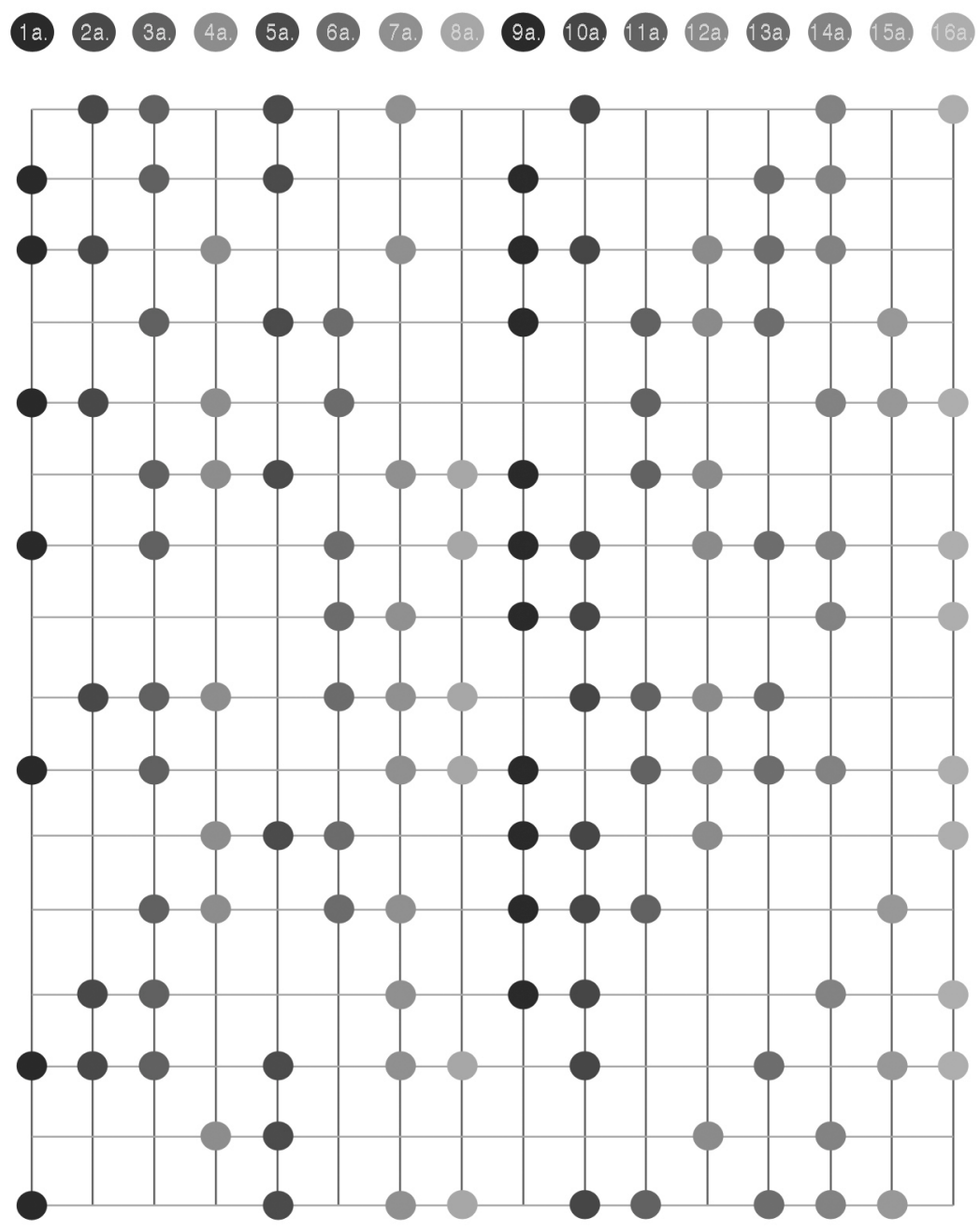

intéractions prédominantes entre thèmes critiques

Fig. 3. Thèmes critiques de l'intégration à niveau urbain : matrice des interactions mutuelles prédominantes. 
cohérence entre les configurations d'ensemble et les parties composantes. L'idée qui a guidé cette analyse critique est en effet liée à la conviction que la qualité de chaque " objet " architectural est étroitement liée au niveau de "consonance " qu'il établit avec son contexte, avec une incidence toujours plus vaste que celle de sa seule dimension spatiale. Cela est confirmé par certaines expérimentations internationales, qui soutiennent une perspective de travail multi-échelles, capable d'exploiter le potentiel des lieux à travers le dépassement d'une vision strictement scalaire.

L'analyse de ces expériences suggère de concentrer I'attention sur ces tendances récurrentes :

- lecture critique de la complexité des caractères du contexte, en tant que base d'interprétation de ses nécessités, ses potentialités et ses limites ;

- augmentation de l'intensité et de la vitalité de la ville, soit par rapport à la densité et à la compacité du bâti ${ }^{10}$, soit par rapport à la mixité fonctionnelle et sociale des espaces, en tant que base d'une forme de démocratie écologique du milieu urbain ;

- valorisation de la diversité des lieux, comme principe de sauvegarde des identités locales, en opposition à la répétition de solutions indifférenciées par rapport aux contextes ;

- exploitation des potentialités des éléments verts et de l'environnement naturel, afin d'assurer la continuité des trames urbaines, I'articulation du paysage, la qualité du rapport entre espace public et privé et la protection de la biodiversité ;

- valorisation des " espaces intermédiaires », en tant qu'instruments clés du contrôle des relations sociales des individus, des interactions physiques-environnementales des lieux et des flux énergétiques entre les bâtiments ;

- renforcement de la perméabilité des espaces et du rôle des interconnexions du système urbain, soit dans l'augmentation des opportunités d'échange, soit dans la diversification des moyens disponibles ;

-renforcement de la lisibilité spatiale, avec une caractérisation plus consciente du rapport entre bâti et espaces ouverts, utile pour faciliter les mouvements et favoriser le « sens de l'appartenance » des personnes aux lieux;

- évolution typologique, morphologique et technologique de modèles et composants de l'architecture, en tant que traduction des exigences liées aux mutations économiques, environnementales et sociales de la réalité actuelle ;

- conception des formes (urbaines et architecturales) résilientes, capables d'absorber facilement les changements relatifs à l'évolution des dynamiques temporelles ${ }^{11}$;

- évaluation de la pertinence des choix de conception et construction, par rapport à la réduction de l'impact environnemental, par rapport à l'utilisation rationnelle des ressources, à la durabilité de l'intervention et au confort sensoriel des usagers ;

- contrôle et vérification ex ante de l'efficacité des stratégies et des actions du projet, à travers des processus de coordination ouverts et dynamiques.

\section{Projet urbain et perspective systémique} La révision de méthodologies obsolètes et le dépassement d'analyses restreintes à une vision scalaire ou sectorielle, représentent les conditions nécessaires
10. Katie Williams, Elizabeth Burton, Mike Jenks, The Compact city: a sustainable urban form? Oxford, Oxford Brookes University, 1996.
11. Randolph T. Ester, Design for Ecological Democracy, Cambridge (Mass.), The MIT Press, 2006. 
pour fonder une approche intégrée du projet, capable d'améliorer la cohérence entre le " tout " et ses "parties ", indépendamment des dimensions d'intervention. Les considérations précédentes ne doivent pas être associées à des échelles strictement quantifiables: c'est-à-dire que pour comprendre la nature et la quantité des forces d'interaction incidentes dans un projet, il faut savoir reconnaître d'abord le degré de complexité spécifique de son contexte. Cette approche peut être rapportée aux concepts de la vision systémique, qui suggère de déplacer l'attention des objets aux relations, en s'opposant à la tendance fréquente de réduire l'évaluation de la qualité à des phénomènes mesurables et décomposables. Ce que le physicien systémique contemporain, Fritjof Capra, soutient (" les systèmes vivants sont totalités intégrées, dont les propriétés ne peuvent pas être réduites à celles de plus petites pièces composantes ») peut et doit être considéré également pertinent pour les systèmes urbains. Le même Capra, dans son livre The Web of Life ${ }^{12}$, affirme que "dans le passage de la pensée mécaniciste à la pensée systémique, la relation entre "parties" et "tout" a été inversée. Dans le contexte de la science cartésienne, on pensait que, dans chaque système complexe, le comportement du "tout" pouvait être analysé en fonction des propriétés de ses parties. La science systémique démontre, au contraire, que les systèmes vivants ne peuvent pas être compris seulement par l'analyse ". En transposant ces considérations aux processus qui règlent la construction du système urbain, on peut dire alors que si dans la vision mécanistique-fonction- naliste la ville se compose de "parties " dont les rapports sont de type essentiellement déterministes, dans la perspective systémique les relations au sein de chaque " objet » ont une importance secondaire, car chacun d'eux constitue un réseau de relations, inséré dans des réseaux encore plus grands. Et l'intérêt des débats internationaux les plus récents sur le thème du " réseau ${ }^{13}$ " confirme l'importance de la diversification et de l'intensification des flux, en tant que bases essentielles de la qualité du développement des établissements humains : le caractère de ces relations matérielles et immatérielles - de la conception aux usages finaux - conditionne en effet l'intégrité du système urbain, la cohérence de sa structure et la fertilité de son potentiel.

L'intégration des facteurs (environnementaux, technologiques, socioculturels et économiques) et des éléments (naturels et artificiels), ainsi que des instruments et des compétences associés à la transformation de l'espace urbain, concerne - à des manières et à des temps différents - tous les acteurs impliqués dans ce processus (politiques, concepteurs, clients et usagers). Cela est lié non seulement aux phases de conception et construction, mais aussi à l'influence que les transformations produisent sur les attitudes des personnes et sur les modalités d'utilisation. L'importance que jouent la maintenance, la gestion et, plus généralement, la modification apportée par les personnes exige alors de considérer les paramètres du temps et des usages, en tant que facteurs de référence d'un projet intégré. Cela signifie qu'il faut adopter de nouvelles perspectives capables de concilier des approches de type :
12. Fritjof Capra, The Web of Life, New York, Anchor Books, 1996.
13. Manuel Castells, La città delle reti, Venise, Marsilio, 2004. 
- multidisciplinaire, pour comprendre la culture du contexte et de ses métamorphoses, dans l'intersection des dimensions spatiales, socioculturelles, environnementales et économiques ;

- multi-scalaire, pour promouvoir la convergence des différents niveaux d'intervention, à travers l'interaction efficace de produits, acteurs et compétences liés à la conception et à la construction de l'espace urbain ;

- temporel, pour définir formes urbaines et architecturales flexibles, par rapport à l'évolution de l'impact de l'action humaine sur l'environnement, à l'incidence des conditions d'utilisation des usagers, et à l'égard des contraintes spécifiques de tous les acteurs impliqués.

Le projet urbain assume aujourd'hui - peut-être comme jamais auparavant - un rôle fondamental en tant qu'instrument d'interprétation de la réalité et de définition des équilibres futurs: déjà reconnu comme une extraordinaire occasion de régénération et requalification du tissu existant, il peut constituer la dimension privilégiée à travers laquelle comprendre, composer et construire les "contenus " et les " contenants" de la ville contemporaine, dans la pluralité des «temps» et des « voix » qui la caractérisent. Les matrices proposées - certainement ouvertes à des développements successifs - ne sont pas conçues pour produire des jugements d'évaluation synthétique, mais plutôt pour induire des réflexions sur l'incidence que les facteurs du projet et des processus ont sur la nature et la qualité des transformations. La lecture de ces "mosaïques" et l'interprétation des conséquences relatives à la simultanéité des relations prédominantes suggère de retrouver le « sens » du projet, en tant qu'expres- sion des potentialités spécifiques d'un espace urbain et référence culturelle essentielle de la société qui l'a produit. La valeur des opérations exemplaires ne peut pas être restreinte à une plus forte qualité environnementale et/ou à une meilleure efficacité énergétique des bâtiments : il s'agit plutôt de la reconnaître dans le mûrissement d'une dimension culturelle plus profonde du projet et d'un sens d'appartenance plus favorable des personnes aux principes soutenus et aux lieux où ils ont pris forme. S'interroger sur la signification que l'intégration assume par rapport à la valeur des transformations urbaines signifie alors atteindre une conscience plus profonde :

- de l'incidence des effets induits par la pluralité de relations mutuelles en jeu ;

- de la responsabilité des comportements sociaux liés à la production et à l'usage des espaces ;

- du potentiel des lieux, relatif à la complexité de chaque contexte.

Chercher des "recettes » ou des règles générales n'est pas la voie à suivre: la singularité de chaque lieu - fruit de la combinaison de caractères particuliers et uniques - exige plutôt "yeux » et " mains » à chaque fois renouvelés, capables de définir les actions et les processus les plus opportuns pour chaque contexte opérationnel. Seule l'évolution d'un imaginaire mental conscient des potentialités des interactions spatiotemporelles associées à la transformation urbaine pourra devenir le "moteur » d'une créativité qui " sachant regarder dans l'obscurité » (Marguerite Yourcenar), saura écrire et préfigurer de nouvelles " pièces » de l'histoire des lieux. 